\title{
Performance Comparison of AOMDV and SMR Protocols for Scalable Video Communication in MANET
}

\author{
Yongzhi Wang* \\ College of Computer Science, Hunan Institute of Science \& Technology, Yueyang, Hunan, 414000, P.R. China
}

\begin{abstract}
With the development of wireless network technology and multimedia technology, multimedia applications of MANET have gained more and more attention. How to ensure the QoS of video communication in multi-hop network is an urgent key problem. Multi-path streaming is a useful way to improve the quality of wireless video transmission. In this paper, we first analyze the routing mechanism of AOMDV and SMR. Then we use NS2 to generate different scenario files using different movement modes, and through forwarding scalable video contents, we test the performance of AOMDV and SMR. At last, we evaluate their performance through packet delivery ratio, average end-to-end latency and normalized routing overhead.
\end{abstract}

Keywords: AOMDV, mobile ad hoc network, scalable video coding, SMR.

\section{INTRODUCTION}

A mobile ad-hoc network (MANET) is an immediately deployable wireless network needing no base station or infrastructure to uphold routing and network management. A MANET includes wireless mobile nodes which are collected and they act as two roles of sending or receiving terminal and router. The nodes are suitable for all the tasks which are required consisting of routing to communicate. Due to the limited transmission range of the nodes, they usually need multiple hops to exchange information with other nodes. Therefore, the routing protocol used in a MANET can directly affect the overall performance of data delivery [1].

With the development of wireless network technology and multimedia technology, multimedia applications of MANET have gained more and more attention. How to ensure the QoS of video communication in multi-hop network is an urgent key problem [2,3]. Multi-path streaming is a useful way to improve the quality of wireless video transmission. Because transmission bandwidth is better than a single path and for the reliability of multiple routes, it can decrease the influence of quality of the video transmission path from the path damage through constructing multiple transmission routes between the source nodes and the destination nodes. There is a substantial study of multi-path routing protocols at present, especially research of on-demand multipath routing protocols which is a hot issue in MANET field [4]. AOMDV [5] and SMR [6] are two classic ondemand multipath routing protocols. In this paper, we will first analyze the routing mechanism of AOMDV and SMR. Then we will use NS2 to generate different scenario files with varying movement patterns, through forwarding scalable video contents, and test the performance of AOMDV and SMR. At last, we are going to evaluate their performance through packet delivery ratio, average end-to-end latency and normalized routing overhead.

\section{ROUTING MECHANISMS OF AOMDV AND SMR}

\subsection{AOMDV Protocol}

AOMDV (Ad Hoc On-demand Multi-path Distance Vector) protocol extends the AODV protocol for calculating multiple loop-free and link-disjoint routes. Routing table entry structure of AOMDV protocol is shown in Fig. (1). It inherits the behavior of AODV protocol that using "destination sequence number" as routing update markers, while adds a new entry called "advertised hop count" to calculate multipath. The sequence number of all the next hops are the same. For each destination, there are a range of the next-hops with the corresponding hop counts to sustain track of multiple routes in the routing entries. In the AOMDV protocol, advertised hop count is defined by the highest hop count for all the routes. When a node's hop count is lower than the advertised hop count, it only receives an alternate route to that goal. Conversely, a routing table containing routing information used recently will be maintained by each node. The routing information includes a next hop and other control information. If a route advertisement is accepted for a destination node with a larger sequence number, the nexthop list and the advertised hop count will be reinitialized.

AOMDV can be utilized for seeking multiple linkdisjoint and node-disjoint paths. What is similar to AODV is that AOMDV employs Route request (RREQ), Route reply (RREP), and Route Error (RERR) to create and maintain its routing information. To find multiple link-disjoint paths, the destination responds to duplicate RREQs without regard to their first hop. In an attempt to ensure getting linkdisjointness routes, only these RREQs arriving through 


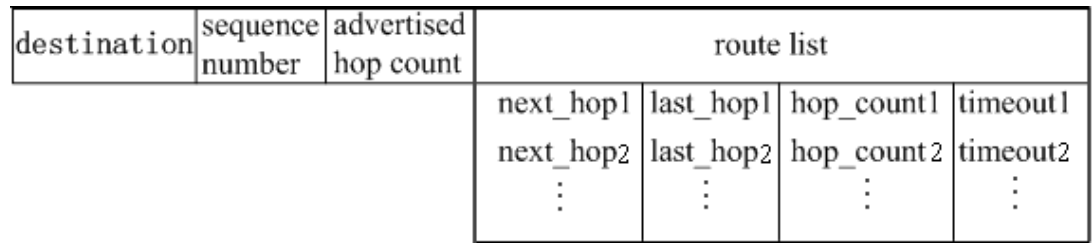

Fig. (1). Routing table entry structure in AOMDV.

unique neighbors are replied by the destination node. Alternate routes to the destination are defined by each duplicate path advertisement received by certain node. AOMDV picks a shortest latency path as the primary route, and another route as an alternate path. Once the primary path fails, the alternate path in route list will be used for transmission as the primary path. In Case a node becomes aware of that all routes through a neighboring node are no longer valid, it will send RERR about the detected error to its neighbors.

\subsection{SMR Protocol}

SMR (Split Multi-path Routing) protocol extends dynamic source routing (DSR) protocol. It is a classic ondemand multipath source routing protocol. In SMR, the full route is contained in the header of the date packet. RREQ and RREP are used to establish maximally disjoint paths between source and destination. Unlike AOMDV, the whole route between source-destination node pairs is contained of in the packet header, and forwarding nodes do not rely on routing tables therefore. Intermediate nodes do not reply to RREQs and not keep a path cache. Thus the destination node can get all the paths. To improve the validity of paths in the route cache of source node, SMR does not allow intermediate nodes sending RREPs to the source node in any case. SMR is not to simply discard duplicate RREQs, but to choose whether to send RREQs according to their hops. Usually SMR only selects two independent routes to send packets. If a destination node receives the first RREQ packet, the shortest delay path will be represented. The destination node can receive more RREQ packets. From these received RREQ packets, the destination selects another maximally link-disjoint path with the shortest latency, and then sends an RREP message to the source for the picked RREQ. In the case of SMR, there are two kinds of route update mechanisms: (1) SMR-1. If any route to the destination node is invalidated, the source node will perform a route discovery. (2) SMR-2. Only when both paths to the destination node are invalidated, the source node initiates route reconstruction. In this paper, we primarily consider SMR-2.

\section{SIMULATION AND EVALUATION}

\subsection{Simulation Tools}

In this study, we use Network Simulator 2 (NS-2) to generate different scenario files using different movement modes, and through forwarding scalable video contents, we test the performance of AOMDV and SMR routing protocols in communicating scalable video coding (SVC) contents under various network scenarios. H.264 SVC is an extension standard of H.264/AVC standard [7, 8]. Relative to H.264/AVC standard, SVC has significant advantages in coding efficiency and degree of supported scalability. SVC can support the layered encoding of video contents which permits the decoding of video contents at varying special, temporal, and DSNR qualities. SVC can encode video contents into a basic layer and some enhancement layers. In this way, destination nodes are able to recover the video streams based on their needs and capabilities needing for no reencoding and forwarding multiple video contents with different qualities. In this paper, we employ NS-2 with a scalable video evaluation framework. Using NS-2, we first specify the network topology, and select sender and receiver according to the simulation scenario. Then we use MyEvalSVC [9] which is an extension for NS-2 to send and receive the video streams traces. We employ Joint Scalable Video Model (JSVM) [10] to encode the input video. Moreover, during the sending the packets of real video, we use Scalable Video Coding streaming Evaluation Framework (SVEF) [11] to evaluate the performance of recovered scalable video. Using these simulation tools enable a real video to be encoded, packetized, and transformed into a format suitable for transmission over the MANET in NS-2. When the simulation ends, at the receiver node, a trace file generated by SVEF of the video will be created. The trace file is able to be transformed into a format which is compatible with NS-2. The video packets already received by the receiver are listed in the output packet trace file. Therefore, the trace file can be utilized to reproduce the video that takes the losses and delay occurred into consideration during the period of transmitting the video streams.

\subsection{Simulation Experimental}

We build 30 nodes networks in a $800 \mathrm{~m} * 600 \mathrm{~m}$ rectangular field. Initially each node is placed randomly and uniformly in the rectangular field. Simulation parameters are shown in Table 1. Each node in the simulation moves according to "random waypoint" model. Each node starts the simulation movement scenario by remaining static for pause time seconds. After selecting a stochastic destination in the $800 \mathrm{~m} * 600 \mathrm{~m}$ field, the node moves to the destination at a speed lying between 0 and $18 \mathrm{~m} / \mathrm{s}$. Once reaching that target location, the node pauses again for $20 \mathrm{~s}$, chooses another termini, and moves there as formerly described. All nodes repeat this behavior during the simulation.

The experiment employs the foreman video sequence. Source-destination node pairs are selected randomly. Traffic pattern consists of several UDP connections. We use JSVM to encode the video sequence into CIF Format with image dimensions $352 * 288$ and $15 \mathrm{fps}$. The length of the video is 750 frames. Each frame is encapsulated into one or more UDP data packets that is less than or equals to 1024 bytes. In the experiments, we use SVEF to get a packet trace file which assigns the sequence number of video frame to each packet, extract parity frames from the packet trace file se- 
Table 1. Simulation parameters.

\begin{tabular}{|c|c|}
\hline Name & Parameters \\
\hline \hline Space size & $800^{*} 600$ \\
\hline Number of nodes & 30 \\
\hline Simulation time & $500 \mathrm{~s}$ \\
\hline Movement model & Random waypoint \\
\hline Data packet size & $1024 \mathrm{~B}$ \\
\hline MAC & 802.11 \\
\hline RF transmission range & $250 \mathrm{~m}$ \\
\hline
\end{tabular}

quence. Through different routes, the odd frames and even frames can be sent, and the multipath transmission can be realized hence.

Comparing the protocols, we mainly think of the following three performance metrics: (1) Average packet delay-the average latency it takes for each data packet to reach the target nodes; (2) Packet delivery ratio-- the ratio between the video packets correctly received by the destination nodes and the generated by the source nodes; (3) Normalized routing overhead-- the ratio of the number of control packets generated by all nodes to the total number of all forwarded packets.

\subsection{Simulation Results}

We pre-generated 70 varying scenario files with different movement patterns, then ran separately AOMDV and SMR routing protocols against these scenario files. Because each protocol was challenged in a duplicate mobility scenario, the performance of the two protocols could be directly compared. The time of each simulation is 600 seconds, and each data point in the plots represents an average of 5 simulations with same traffic models, but various mobility scenarios are generated randomly. In all the experiments, the highest speed of the nodes in the scenario is varied from 0 to $18 \mathrm{~m} / \mathrm{s}$ to alter mobility.

The average packet delay as a function of varying mobility is reported in Fig. (2). For video communication, a lower delay means a better performance. Fig. (2) shows that SMR protocol has better performance when the speed of nodes is lower than $8 \mathrm{~m} / \mathrm{s}$. However, at higher speed of nodes, AOMDV performs better. At the same time, it is noted in Fig. (2) that there is a decline in average delay values for speed $6 \mathrm{~m} / \mathrm{s}$ from the delay values for speed $4 \mathrm{~m} / \mathrm{s}$. This is because speed affects the routing a little as the nodes move slowly, and it will even increase the number of candidate paths, so that the multipath routing can choose the best path to forward video packets. Otherwise, when the speeds become fast enough, the failure probability of packet transmission will become larger, and frequent path repair process will result in higher delay.

Fig. (3) presents the packet loss behaviors of AOMDV and SMR. Packet delivery ratio refers to the ratio between the video packets correctly received by the destination nodes and the generated by the source nodes. It embodies the reli-

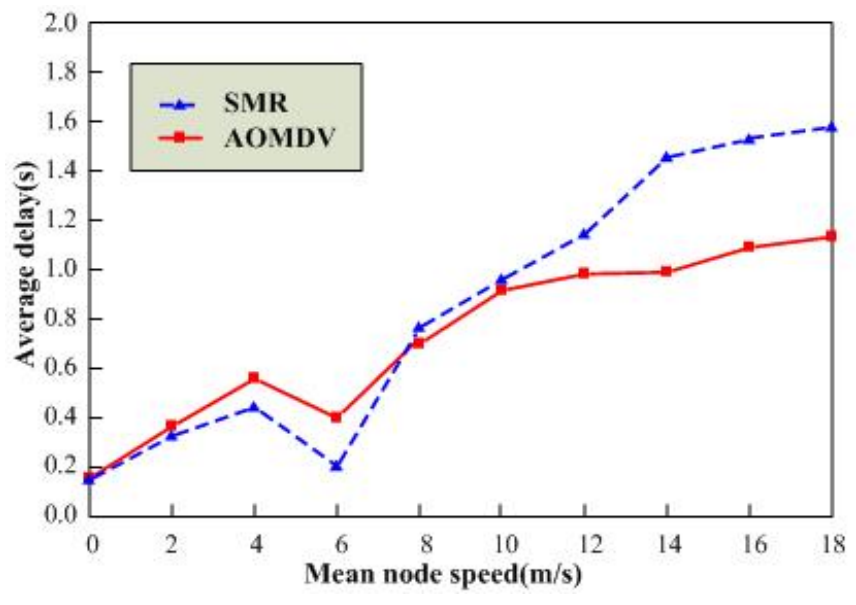

Fig. (2). Average packet delay with varying mobility.

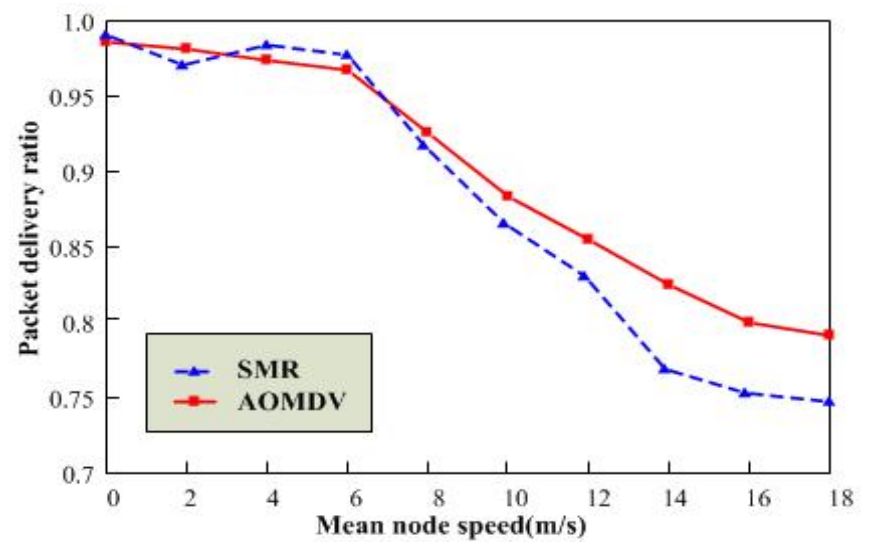

Fig. (3). Packet delivery ratio with varying mobility.

ability of a routing protocol in transferring data from the source to the destination. In real-time applications such as the wireless video communication, when the delay exceeds limits, the received video packets will be considered as lost data packets and will not be used to decode the video frames. We can find it clearly from the Fig. (3) that the percentage of correctly decoded packets decreases with mean node speed. But SMR has slightly lower performance than AOMDV protocol especially in high-speed mobility scenarios. The reason is that SMR uses multiple active paths alternately to forward video packets, when the speeds become fast enough, due to the active paths fail frequently, packets sent through stale paths might either finally be lost or delayed.

Fig. (4) presents the performance comparison of control overhead in normalized routing load between using AOMDV and SMR. Normalized routing overhead refers to the ratio of the number of control packets generated by all nodes to the total number of all forwarded packets. Hence it can reflect the efficiency of a protocol. We find it in Fig. (4) that, the network topology changes acutely as node speed increases, which leads to a larger possibility of link breakage, an increased frequency of route reconstruction, and a significantly increased normalized routing cost with each protocol. Fig. (4) shows that the normalized routing overhead of MSR is a little higher, because SMR allows the destination nodes sending lots of RREPs for the selected maximum disjoint routes, and uses multicast transmission. Par- 
ticularly in high-speed network environments, this multipath and multicast protocol results in a larger probability of packet retransmission failure and the behavior that packets reach the destination nodes through different paths will result in "packet disorder" phenomenon. Furthermore, reassembling the packets will increase transmission overhead.

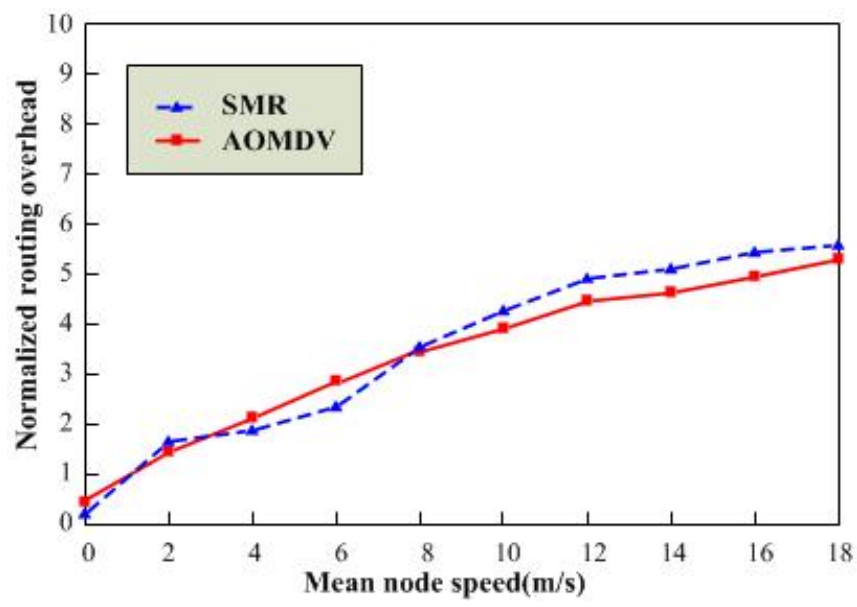

Fig. (4). Comparison of normalized routing overheads.

\section{CONCLUSION}

At present, how to ensure the QoS of video communication in MANET is an urgent key problem. The selection of the multi-path routing protocol can have a significant impact for video transmission quality. In this paper, we use NS2 to build different network topology scenarios with varying movement patterns. Through forwarding scalable video contents, we test the performance of AOMDV and SMR and the Simulation results show that, in low-speed or medium-speed MANETs, SMR protocol has certain advantages in average delay and packet delivery ratio aspects at the expense of larger routing overhead. However, in high-speed MANETs, AOMDV shows better performance and more efficient fault recovery mechanism.

\section{CONFLICT OF INTEREST}

The author confirms that this article content has no conflict of interest.

\section{ACKNOWLEDGEMENTS}

This work was financially supported by the Hunan Province Natural Science Foundation of P.R. China (Grant No. 14JJ4060).

\section{REFERENCES}

[1] H. Zhou, "A Survey on Routing Protocols in Manets," Technical Report: MSU-CSE-03-08, 2003.

[2] X. Zhang, and Z. Guo, "Rate-distortion optimized multi-path selection for video streaming over wireless multi-hop networks", Journal of Software, vol. 22, no. 10, pp. 2412-2424, 2011.

[3] S. Mueller, and D. Ghosal, "Multipath Routing in Mobile Ad Hoc Networks: Issues and Challenges", Lecture Notes in Computer Science, Springer, Berlin, 2004, pp. 209-234.

[4] H. An, and X. Lu, "A review of routing protocols for mobile ad hoc networks," Computer Engineering and Science, vol. 28, no. 2, pp. 4-9, 2006.

[5] M.K. Marina, and S.R. Das, "Ad hoc on-demand multipath distance vector routing," Wireless Communications and Mobile Computing, vol. 6, pp. 969-988, 2006.

[6] S. Lee, and M. Gerla, "Split multi-path routing with maximally disjoint paths in ad hoc networks", Communications, IEEE International Conference on, vol. 10, pp. 3201-3205, 2001.

[7] ITU-T and ISO/IEC JTC1, JVT-W201, "Joint Draft 10 of SVC Amendment", 2007.

[8] M. Halloush, H.R. Al-Zoubi, Z. Al-Qudah, and O. Alkofahi "The Performance of MANET Routing Protocols for Scalable Video Communication," Communications and Network, vol. 5, pp. 119$125,2013$.

[9] C.H. Ke, "myEvalSVC: An Integrated Simulation Framework for Evaluation of H.264/SVC Transmission," KSII Transactions on Internet and Information Systems, vol. 6, no. 1, pp. 378-393, 2012.

[10] J. Vieron, M. Wien and H. Schwarz, "JSVM-9.14 Software, Joint Video Team (JVT) of ISO/IEC MPEG \& ITU-T VCEG,” 2007.

[11] A. Detti, G. Bianchi, C. Pisa, F. S. Proto, P. Loreti, W. Kellerer, S. Thakolsri, and J. Widmer, "SVEF: An open source experimental evaluation framework for H.264 scalable video streaming," In: IEEE Symposium on Computers and Communications, Sousse, 2009, pp. 36-41.

Received: June 10, 2015

Revised: July 29, 2015

Accepted: August 15, 2015

(C) Yongzhi Wang; Licensee Bentham Open.

This is an open access article licensed under the terms of the Creative Commons Attribution Non-Commercial License (http://creativecommons.org/licenses/by-nc/3.0/) which permits unrestricted, non-commercial use, distribution and reproduction in any medium, provided the work is properly cited. 